\title{
Connecting the Dots: Links between Kinetic Theory and Bernoulli's Principle
}

\author{
Katherine Misaiko* and James Vesenka* \\ *Department of Chemistry and Physics, University of New England, 11 Hills Beach Road, Biddeford, ME 04005
}

\begin{abstract}
Kinetic Theory and Bernoulli's Principle are fundamental concepts life science students can use to explain a variety of important biological phenomena. Based on paired student interviews we established that many UNE students had no molecular models to describe fluid behavior, and what models students did have were typically flawed. We developed simple experimental activities and questions to help pinpoint student preconceptions regarding ideal gas behavior and a fluid dynamics concept relating to Bernoulli's Principle. Students were asked to use multiple representations (diagrams, graphs, math and verbal descriptions) to explain these experiments. Our research suggested that understanding Bernoulli's Principle was helped by having a conceptual understanding of kinetic theory, in particular equating pressure with particle collisions. Based on these results a multi-representational "modeling centered" ideal gas law lab, using semi-quantitative diagrammatic tools, was created. We tested the lab on our spring 2013 general physics students in order to investigate the impact of the improved models on Bernoulli's Principle comprehension. The same students were post-tested on a new set of similar ideal gas and fluids dynamics problems with the results indicating substantial improved gains in answering fluids statics questions and modest gains on Bernoulli Principle questions.
\end{abstract}

Keywords: Multiple representations, particle model, Bernoulli Principle, Ideal Gas Law, kinetic theory PACS: 01.40.Fk, 01.40.G-

\section{INTRODUCTION}

The most common approach to developing the Bernoulli Equation is through conservation of energy:

$$
\Delta P=-\frac{1}{2} \rho \Delta\left(v^{2}\right)-\rho g \Delta y
$$

Pressure "P" is the fluid's energy density, " $\rho$ " is the fluid's density, "v" relates to the fluid's speeds, "g" is gravitational field and "y" is the elevation of the fluid. The right hand side of the equation describes the pressure change due to kinetic (dynamic) and gravitational energy density (hydrostatic pressure) differences. The problem with this mathematical representation is that students have little conceptual understanding of the physical processes taking place. Furthermore the Bernoulli Principle is often incorrectly used to explain many fluid dynamics phenomena [1],[2]. Still there are important physiological conditions in which the Bernoulli Principle can be used to conceptually predict fluid behavior such as atherosclerosis or an aneurysm. The research described here resulted in identifying the need for better diagrammatic tools, deployment of a new fluids statics lab, and evaluating the students' understanding through both an existing assessment and one new set of questions based on student-generated distractors.

\section{Local context}

The Physics Education Research Groups of the University of New Hampshire and University of New England (UNE) have been collaborating for the past six years on investigating student understanding of fluid mechanics. The emphasis on fluids is driven by developing curriculum tuned to the needs of life science majors, which represent $>95 \%$ of our physics students. The fluids content was informed by physical science competencies described in the HHMI/AAMC 2009 report [3]. At UNE physics is delivered in studio classrooms (24 students in a combined lecture-lab) using modeling instruction [4] emphasizing graphical and diagrammatic representations. We have made progress developing modeling-centered labs for buoyancy [5], fluid drag, and viscosity [6] in the context of introductory physics for life science students [7]. Our research focused on determining student preconceptions of fluid behavior based on student-pair interviews at UNE. 17 students participated in the research in the summer of 2012, and 34 students participated in the spring of 2013. The interviews were conducted before and after classroom exposure to our fluids curriculum consisting of five laboratory experiments including buoyancy, The Bernoulli Principle (2 labs), fluid drag and viscous drag. The spring 2013 students participated in a new ideal gas lab motivated by the preliminary results of this study. 


\section{Research Problem}

We were interested in determining what, if any, models students might have to explain the Bernoulli Principle. We investigated this question by having the 2012 students explain the two demonstrations described in Fig. 1. The first demonstration was a fluid statics problem that we thought would be easy to explain because all students in our study previously had completed general chemistry in which the ideal gas law was covered. The second demonstration was a fluid dynamics problem that yielded an identical observation, but for different mechanical reasons.

\section{INTERVIEW RESULTS}

\section{Pre-Fluids Coverage}

About half our pre-interview groups were unable to provide any graphical or mathematical explanation for the pressure change in Figs. 1(a\&b). Fewer still used molecular models to explain the behavior of either demonstration. None of the students could explain the behavior of the manometer when an air stream was directed past the tube's end in Figs. 1(c\&d).

During the pre-interviews most students who were



FIGURE 1. a) Dye filled plastic manometer at equilibrium, left side open to atmosphere, right side connected to sealed air filled glass jar. b) Visible pressure reduction in right arm after cooling the jar in blue liquid. c) Same manometer but open on both ends with streamer attached to right side. d) Air blown obliquely past the tube opening generates lower pressure, with air motion indicated by the streamer.

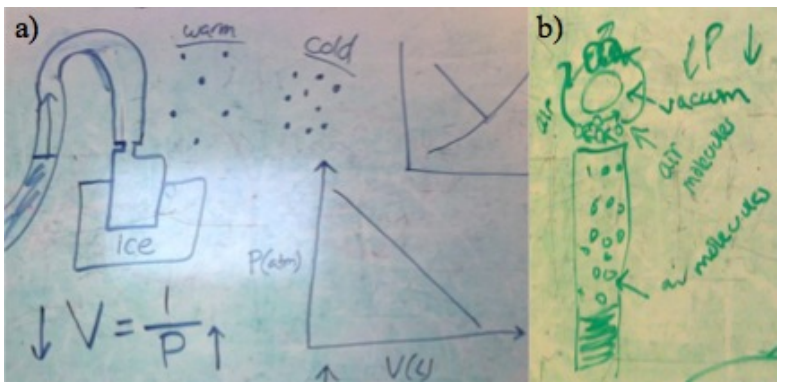

FIGURE 2. a) Student explanations of pressure change described in Fig. 1(b), ccw from bottom left. The students mistakenly claimed increased pressure leads to decreased volume when temperature decreased. Weak math skills are common: inverse drawn as a straight line with negative slope. Lastly, a particle model diagram was attempted to explain the pressure decrease when the molecules cool down, confusing pressure with particle density. b) Student diagram attempting to explain Fig. 1(d) with air molecules avoiding the vacuum.

unsuccessful at explaining the observations had little understanding of the ideal gas law. We noted the following skills deficiencies or preconceptions in common (one interesting explanation noted):

- Could not draw the graph of an inverse relationship, bottom right Fig. 2(a).

- Could not distinguish the difference between particle behavior and number of particles.

- Thought particles moved from areas of low concentration to areas of higher concentration.

- Equated pressure with density, top center Fig. 2(a).

- Thought vacuums "suck" or "pull" fluids.

- One group thought that the air molecules "move around the vacuum", Fig. 2(b).

The few groups that were successful at explaining the experimental observations all had sound math skills and good comprehension of the ideal gas equation. They also had the following in common:

- Made unprompted use of particle models.

- Understood the difference between particle motion and number.

During the interviews we discovered the majority of students neither understood, nor could model, the concept of "pressure". Those who responded "force/area" were asked to explain what this definition meant to them. Only $21 \%$ of the interviewees offered microscopic or molecular explanations. These findings are consistent with research on microscopic properties of fluids conducted elsewhere [8],[9].

\section{Post-Fluids Interviews}

Post-interviews were taken after our students completed the fluids units. We found the following:

- Some weak students transitioned to using microscopic particle models in their explanations. 


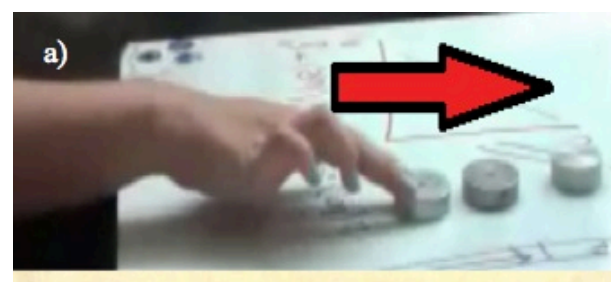

b)

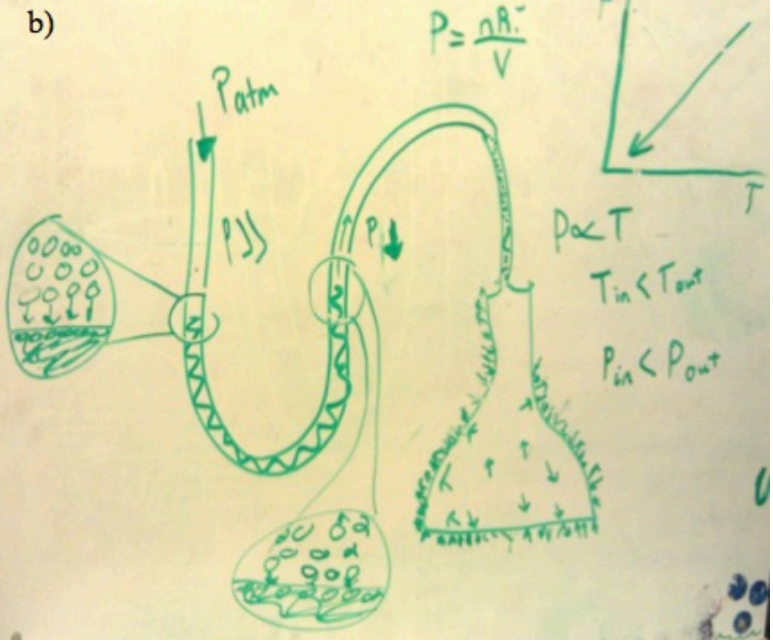

FIGURE 3. a) Prompting students to employ a particle model using hard spheres to explain that pressure is the result of pushing. b) Post-content coverage white-board explaining Fig. 1(b). Note the student drew pressure pushing the fluid and made correct math, graphical, and diagrammatic models.

- Those that did not, with prompting shown in Fig. 3(a), could use a particle model to explain the observed ideal gas behavior.

- Changed explanation from pressure pulling the water up the tube to pushing the water from the other side shown in Fig. 3(b) and enumerated in Table I.

A summary of pre/post interview results from 2012 $(\mathrm{N}=17)$ and $2013(\mathrm{~N}=34)$ is found on Table I. We concluded that constructing understanding of a conceptual microscopic "particle model" would help improve student understanding of fluid static pressure. Since students had no better understanding of pressure in fluid dynamics we suspected it might also improve understanding of the Bernoulli Principle.

TABLE I. Pressure Deficiencies

\begin{tabular}{l|c|c|c}
\hline Preconception or Skill & Year & Pre & Post \\
\hline Vacuums pull or suck & 2012 & $53 \%$ & $10 \%$ \\
\hline $\begin{array}{l}\text { Particles move from high } \\
\text { to low concentration. }\end{array}$ & 2012 & $42 \%$ & $0 \%$ \\
\hline $\begin{array}{l}\text { Particle explanation of } \\
\text { Ideal Gas Activity }\end{array}$ & 2012 & $21 \%$ & $31 \%$ \\
\hline $\begin{array}{l}\text { Particle explanation of } \\
\text { Ideal Gas Activity }\end{array}$ & 2013 & $55 \%$ & $88 \%$ \\
\hline $\begin{array}{l}\text { Particle explanation of } \\
\text { Bernoulli Activity }\end{array}$ & 2013 & $12 \%$ & $18 \%$ \\
\hline
\end{tabular}

\section{CURRICULAR CHANGE}

\section{Introducing The Ideal Gas Law}

Though the target of our research was to improve understanding of the Bernoulli Principle, the research outcome indicated students needed help developing microscopic models of describing pressure to be able to appreciate the cause of pressure differences. The existing suite of fluid lab activities appeared to have limited impact at improving our 2012 students' abilities to explain pressure as seen in Table I. We incorporated the research results into a new particle-based ideal gas laboratory delivered in the spring of 2013. Diagrammatic representations of the ideal gas law played a key role in the discussion activities.

$$
P V=N k_{B} T
$$

Where $\mathrm{P}=$ pressure, $\mathrm{V}=$ volume of gas, $\mathrm{N}=$ particle number, $\mathrm{k}_{\mathrm{B}}=$ Boltzmann's constant and $\mathrm{T}=$ temperature of the ideal gas. Though the ideal gas equation describes pressure as the result of noninteracting particles only colliding with the container walls we included collisions between gas particles. Diagrammatically volume was treated as a container size and the number of particles simply as the number of arrows. Temperature was treated as randomly oriented arrows with shorter lengths for lower energy particles and longer lengths for higher energy particles. Pressure was treated as the number of interactions between the particles and container or other particles ${ }^{1}$.

\section{Post-Fluids Interviews and Assessment Results}

With new diagrammatic tools in place we undertook interviews after coverage of the ideal gas lab during the spring 2013 semester. Students dramatically improved their ability to explain ideal gas experiments, activities that were different from demonstrations used in class. The unprompted use of particle models to describe ideal gasses increased to $88 \%$ of the participants compared to $31 \%$ in 2012 . Based on the interview results we added to a distractorbased fluids assessment four new multiple-choice questions (two ideal gas and two Bernoulli Principle concepts) to assess the effectiveness of the curriculum change. The new questions came in pairs and consisted of both graphical and diagrammatic problems. The post-test and normalized gain results

\footnotetext{
1 The lab activity, workbook readings and problems are at: http://faculty.une.edu/cas/jvesenka/scholarship/index.htm
} 
TABLE II. Ideal Gas/Bernoulli Correct Answers

\begin{tabular}{l|c|c}
\hline Question & Post (\%) & nGain (\%) \\
\hline Graphical Ideal Gas & $40 \%$ & $34 \%$ \\
\hline Diagrammatic Ideal Gas & $63 \%$ & $45 \%$ \\
\hline Graphical Bernoulli & $25 \%$ & N.A. \\
\hline Diagrammatic Bernoulli & $24 \%$ & N.A. \\
\hline
\end{tabular}

consisted of both graphical and diagrammatic problems (Table II). The complete distractor-based assessment is available online at footnote 1. The assessments results were promising. The students' had realized solid normalized gains for the ideal gas questions. Though the graphical ideal gas question only had $40 \%$ correct on the post-test, it included multiple correct choices. $86 \%$ of the students had at least one of the correct choices. The results from the Bernoulli questions at first glance appear discouraging, but even they have some silver linings.

\section{The Link to Bernoulli}

We made time in our fluids curriculum for the ideal gas lab by converting our quantitative Bernoulli lab to an interactive lab demonstration. Students were still responsible for the same fluid dynamics problems as covered in previous years. Even though we reduced the coverage of the Bernoulli Principle (BP) in class we saw modest improvements regarding correct explanations of BP in the student interviews and assessment results. The post interviews (Table I) indicated incremental additional use of microscopic models to explain the pressure drop in our chief BP demonstration apparatus, the Venturi tube. The silver lining in the graphical Bernoulli question is that even though only a quarter of the class chose the correct answer, $44 \%$ of the class selected one of two pressuredecreasing graphs. The positive spin on the diagrammatic Bernoulli question is that the same distractor question had been used in a general fluids assessment in previous years. The correct response rate had nearly doubled. Furthermore, debriefing of the four UNE instructors after the semester was over indicated weak understanding of the microscopic fluid dynamics models, an oversight by this paper's senior author. The upshot was that even though we reduced quantitative coverage of the BP, we still had a small increase use of microscopic models from interviewees and saw improvements from the general physics population in the two fluid dynamics questions. Indeed, our general fluids pre/post test (no ideal gas questions) annually given to all physics students yielded a $47 \%$ normalized gain in the spring of 2013, 20 points higher than the previous five-year average.

\footnotetext{
${ }^{2}$ Post $=$ Post test score, Pre $=$ Pre test score

${ }^{3}$ Normalized gain defined as (Post-Pre) $/(100 \%$-Pre)
}

\section{CONCLUSIONS}

The interview results indicated approximately half our general physics population had no conceptual picture for describing pressure, despite having previously covered the topic extensively in general chemistry. We changed our curriculum to develop a modeling-centered ideal gas laboratory sequence, complete with diagrammatic models and associated workbook activities based on multiple particle collisions. A multiple-choice assessment based on student-generated distractors and a second round of interviews were used to evaluate student comprehension of the new curriculum. We found that a particle model was helpful in providing students with a conceptual framework for describing fluid statics problems. We also garnered substantial improvements in our annual fluids assessment. Even though we reduced fluids dynamics coverage the new curriculum appears to have provided some benefit to student understanding of the Bernoulli Principle. Our next step is to improve training of our instructors in guided inquiry of the particle models in their classes. Future work is concentrating on developing a consistent microscopic liquid model, similar to the microscopic ideal gas particle model, to help explain the pressure decrease observed in a Venturi tube.

\section{ACKNOWLEDGMENTS}

Supported provided by NSF through DUE grant numbers 0737458,1044154 and the University of New England summer undergraduate student research program.

\section{REFERENCES}

1. K. Weltner and M. Ingelman-Sundberg (unpublished)

2. P. Eastwell, Sci. Ed. Rev. 6, 1-13 (2007).

3. Howard Hughes Medical Institute and American Association of Medical Colleges "Scientific Foundations for Future Physicians" AAMC, Washington, DC (2009).

4. M. Wells, D. Hestenes and G. Swackhamer. Am. J. Phys. 63, 606-619 (1995).

5. M. van den Berg, J. Olivera, M. Weglarz, and J. Vesenka; (to be published).

6. D. Meredith, and J. Bolker, Am. J. Phys. 80, 913-922 (2012)

7. C. Crouch, R. Hilborn, S.A. Kane, T. McKay, and M. Reeves, APS News, 10(3), 8 (2010).

8. C.H. Kautz, P.R.L. Heron, P.S. Shaffer, \& L.C. McDermott, Am. J. Phys. 73(11) 1064-1071 (2005).

9. A.D. Robertson and P.S. Shaffer, Am. J. Phys. 81, 303$312(2013)$. 